\title{
CT colonography's role in the COVID-19 pandemic: a safe(r), socially distanced total colon examination
}

\author{
Courtney C. Moreno ${ }^{1}$. Judy Yee ${ }^{2}$. Firas S. Ahmed ${ }^{3} \cdot$ Matthew A. Barish $^{4}$. Cecelia Brewington ${ }^{5}$. \\ Abraham H. Dachman ${ }^{6}$. Marc J. Gollub ${ }^{7}$. David H. Kim ${ }^{8}$. Elizabeth McFarland ${ }^{9}$. Perry J. Pickhardt ${ }^{8} \cdot$ Syam Reddy $^{10}$. \\ Michael Zalis ${ }^{11} \cdot$ Kevin J. Chang ${ }^{12}$
}

Received: 30 May 2020 / Revised: 12 July 2020 / Accepted: 18 July 2020 / Published online: 3 August 2020

(c) Springer Science+Business Media, LLC, part of Springer Nature 2020

\begin{abstract}
Purpose To describe the favorable procedural profile of CT colonography (CTC) during the COVID-19 pandemic.

Conclusion Postponement of cancer screening due to COVID-19 has resulted in a backlog of individuals needing to undergo structural examination of the colon. The experience during the initial COVID-19 surge with urgent evaluation of the colon for transplant patients prior to transplant suggests that CTC can be done in a lower risk manner as compared to other structural examinations. The procedural profile of CTC is advantageous during this pandemic as maintaining social distancing and preserving healthcare supplies including PPE are of paramount importance. CTC is an important option to utilize in the screening armamentarium to allow effective screening of average risk asymptomatic individuals in the COVID-19 era.
\end{abstract}

Courtney C. Moreno

courtney.moreno@emoryhealthcare.org

Judy Yee

jyee@montefiore.org

Firas S. Ahmed

f.ahmed05@fulbrightmail.org

Matthew A. Barish

MBarish@ northwell.edu

Cecelia Brewington

cecelia.brewington@utsouthwestern.edu

Abraham H. Dachman

adachman@radiology.bsd.uchicago.edu

Marc J. Gollub

gollubm@MSKCC.ORG

David H. Kim

DKim@uwhealth.org

Elizabeth McFarland mcfarlandb123@gmail.com

Perry J. Pickhardt

PPickhardt2@uwhealth.org

Syam Reddy

sreddy.imaging@gmail.com

Michael Zalis

mzalis@mgh.harvard.edu

Kevin J. Chang

kevin.j.chang@gmail.com
1 Department of Radiology and Imaging Sciences, Emory University, Atlanta, GA, USA

2 Department of Radiology, Montefiore Health System and Albert Einstein College of Medicine, Bronx, NY, USA

3 Department of Radiology, Columbia University Medical Center, New York, NY, USA

4 Department of Radiology, Northwell Health, Manhasset, NY, USA

5 Department of Radiology, University of Texas Southwestern Medical Center, Dallas, TX, USA

6 Department of Radiology, University of Chicago, Chicago, IL, USA

7 Department of Radiology, Memorial Sloan Kettering Cancer Center, New York, NY, USA

8 Department of Radiology, University of Wisconsin School of Medicine and Public Health, Madison, WI, USA

9 Department of Radiology, SSM Health, Lake St. Louis, MO, USA

10 Radiology Partners, University of Chicago Ingalls Memorial Community Hospital, Harvey, IL, USA

11 Department of Radiology, Massachusetts General Hospital, Boston, MA, USA

12 Department of Radiology, Boston University School of Medicine, Boston, MA, USA 
Keywords CT colonography $\cdot$ COVID-19 $\cdot$ Colorectal cancer $\cdot$ Colorectal cancer screening $\cdot$ CTC $\cdot$ Pandemic

\section{Introduction}

One of the many unavoidable but potentially life-altering consequences of the current COVID-19 pandemic is the postponement of cancer screening. Colorectal cancer screening has dropped $86 \%$ during the pandemic relative to averages prior to January 20, 2020, according to data from the Epic Health Research Network [1]. Guidance from the Centers for Disease Control and Prevention and directives from state officials resulted in the postponement of elective procedures including cancer screening at facilities to prevent community spread and to preserve the healthcare workforce, facilities, and personal protective equipment (PPE) for an anticipated surge of COVID-19 patients [2]. Additionally, the American Cancer Society recommended that individuals should not go to a health care facility for routine cancer screening at the beginning of the pandemic in the USA [3]. As the pandemic has evolved, concerns to handle the surge have eased and a phased reopening has begun in some states with the Centers for Medicare and Medicaid Services noting that "it is important to restart care that is currently being postponed, such as certain procedural care..., chronic disease care, and, ultimately preventive care" [4]. In regions and facilities meeting recommendations for resuming care including adequate facilities, testing, supplies, and workforce, a large backlog of screening exams is anticipated.

Based on currently available information, the USA may not experience a return to a pre-COVID-19 "normal" until an effective vaccine and/or treatment for COVID-19 is available or enough Americans have been exposed to COVID19 such that population level herd immunity develops. It is difficult to estimate when those gating conditions will arise, though as one example, estimates for the scaled roll-out of an effective vaccine are generally on the order of many months to years from the present. In the meantime, the new normal will likely include continued social distancing, contact tracing, and aggressive testing with fluctuating levels of new positive COVID-19 patients including a possible reemergence in the fall/winter of 2020 [5]. Thus, the initial reasons and issues raised by the surge to delay screening remain to a large extent. This paper highlights the experience during the surge and potential solutions to mitigate risk as screening resumes in the upcoming months.

\section{CTC during COVID-19 pandemic}

CT colonography (CTC) for colorectal cancer screening has typically been canceled and deferred to a later date during the initial COVID-19 surge as highlighted by an internal survey of the American College of Radiology's Colon Cancer Committee. All committee members noted that elective CTC screening had ceased at their institutions. The screening situation by CTC mirrors the experience at screening colonoscopy where similar guidance from the American Society of Gastrointestinal Endoscopy suggested that all elective procedures including screening and surveillance colonoscopy in asymptomatic patients be delayed during the current pandemic [6].

CTC, however, has continued to be undertaken as an urgent or semi-urgent test during this time period. Among diagnostic indications, the one main clinical scenario involves screening of patients for colorectal cancer prior to solid organ transplants. This is a primary indication for CTC undertaken during this time at the University of Texas Southwestern Medical Center (Dallas, Texas), Duke University Medical Center (Durham, North Carolina), and The University of Chicago Medical Center (Chicago, Illinois). Here, CTC has been undertaken as a preferred lower risk alternative to optical colonoscopy, allowing for effective assessment and exclusion of significant polyps and cancers prior to transplant without some of the issues associated with colonoscopy.

\section{CTC's favorable procedural profile during the COVID-19 pandemic}

The procedural profile of CTC as a structural examination of the colon is favorable in the COVID-19 era where social distancing and preservation of PPE are prioritized to lessen community spread and preserve medical supplies given the likelihood of a second wave (Table 1). CTC images are acquired by a single healthcare worker (typically a CT technologist) who is briefly in close proximity to the patient while inserting a small flexible catheter a short distance into the rectum. The CT technologist can monitor colonic insufflation while standing $>6$ feet away from the patient and then moves to the CT control room for the remainder of the procedure thus maintaining social distancing. CTC is performed with disposable equipment, the filter on CTC catheters prevents contamination of the insufflator, and technologists have minimal contact with stool when disposing of the tubing at the conclusion of the examination. SARS-CoV-2 viral RNA has been detected in stool samples of patients with COVID-19 infection [7, 8]. Although the risk of spread by fecal aerosolization is unknown, use of appropriate PPE as per institutional policies for possible aerosol generating procedures (example, N95 mask) is suggested to reduce the risk of healthcare worker infection. 
By comparison, optical colonoscopy utilizes more pieces of PPE as it requires more healthcare workers in close proximity to the patient for longer periods of time including the endoscopist and additional healthcare workers such as an anesthesiologist or nurse anesthetist to administer sedation and a nurse to provide procedural and post-procedural monitoring as the patient recovers from sedation. A healthcare worker such as a nurse is also required to place an intravenous line prior to the colonoscopy.

As sedation is not utilized for CT colonography, at the conclusion of the exam (which typically lasts approximately 15-20 min) the patient can exit the radiology department and drive home without need for post-procedural recovery from sedation within a healthcare facility. This results in a very reduced "dwell time" in the imaging facility which contributes to the goal of achieving limited exposure to other people and environments. Some outpatient radiology practices are implementing workflows where patients are able to wait in their car, walk directly from their car to the CT suite upon notification that it is their time to be imaged, and then directly to their car following the completion of the procedure thereby maintaining social distancing and minimizing contact with other patients and healthcare workers. Additionally, the lack of sedation also limits exposure to a family member or friend who would otherwise be needed to drive a patient recovering from sedation home further maximizing social distancing.

Furthermore, the risk of perforation during screening CTC is significantly lower than optical colonoscopy $(0.0019 \%)$ with most patients not requiring surgery [9]. By comparison, the perforation rate during screening optical colonoscopy without polypectomy is $0.056 \%$ and is $0.062-0.082 \%$ for colonoscopy overall $[10,11]$. Though undesirable at any time, a screening procedure-associated perforation is especially undesirable in the COVID-19 era as it would result in unnecessary hospitalization, utilization of healthcare resources, and potential patient exposure to hospitalized COVID-19 patients. In addition to the risks of perforation, the overall hospitalization rate following colonoscopy is $1.6 \%$ within the first 7 days and $2.4-3.8 \%$ within 30 days following the procedure [12].

The referral rate from CTC to optical colonoscopy is 7.9-13.9\%, so for $>85 \%$ of patients, screening ends with a negative CTC exam $[13,14]$. A repeat screening CTC exam in 5 years is recommended for patients with a normal exam [15].

Although stool-based tests such as multitarget stool DNA tests (e.g., Cologuard (Exact Sciences, Madison, Wisconsin)) do not require that the patient travel to a healthcare facility, the ability of stool DNA tests to detect precancerous lesions is lower than optical colonoscopy and CTC. In a study of 9989 patients who underwent optical colonoscopy, multitarget stool DNA tests detected $92.3 \%$ of cancers but only $42.4 \%$ of advanced precancerous lesions (defined as lesions including advanced adenomas and sessile serrated polyps measuring $1 \mathrm{~cm}$ or more), and $17.2 \%$ of nonadvanced adenomas [16]. Additionally, CTC can better triage patients as compared to stool-based tests for polypectomy or surveillance depending on the lesions found. CTC identifies the size and location of lesions, so if just diminutive/small polyp(s) are found during pandemic times, the patient may elect to undergo an interval 2- to 3-year follow-up with CTC or optical colonoscopy based on age, co-morbidities, and symptoms. By comparison, patients with a positive stoolbased test typically proceed to optical colonoscopy. The false positive rate of fecal DNA testing is $10.2-13.4 \%$ [16].

\section{CTC availability and accessibility}

Insurance claims data and data from the American College of Radiology's My CT Colonography locator tool indicate that CTC is available across the USA though utilization is variable and generally low $[17,18]$. As part of the Affordable Care Act of 2010, commercial insurance plans created on or after September 23, 2010, are required to cover screening CTC without cost-sharing by the patient (no deductible, no copayment) based on CTCs A grade from the US Preventive Services Task Force [19, 20]. Medicare currently does not cover CTC for a screening indication but does cover CTC for some diagnostic indications. CTC is less costly than colonoscopy for colorectal cancer screening of commercially insured patients at the population level [21]. As screening CTC is performed without intravenous contrast material, it can be performed at imaging centers without need for onsite contrast coverage as there is no risk of allergic reaction during the procedure.

Individuals looking for a location that performs CTC in their area can utilize the American College of Radiology's My CT Colonography locator tool [18]. This freely available web site allows the end user to enter his/her address or zip code, and institutions performing CTC nearby are displayed. Institutions performing CTC can add their site(s) to this tool at no charge by registering via a brief web form and entering information including site address and phone number.

\section{Gastrointestinal manifestations of COVID-19, implications for CTC screening}

Although symptoms caused by COVID-19 are most often respiratory, patients may also develop gastrointestinal symptoms. The first known case of COVID-19 reported in the USA presented with a persistent dry cough and 2-day history of nausea and vomiting, and both respiratory and 
Table 1 Usefulness of CT Colonography (CTC) during the COVID-19 Pandemic

1. Similar to optical colonoscopy, CTC is a structural preventative exam and can see the entire colon.

2. CTC has a lower risk of colon complications such as perforation as compared to optical colonoscopy with lower likelihood of requiring an inpatient bed during the pandemic.

3. CTC is performed without sedation so there is no need for another person to accompany or drive the patient to the imaging center thus promoting social distancing.

4. No risk of an adverse reaction to anesthetic with resultant need for hospitalization during the pandemic and sedative medications are preserved for other patients.

5. CTC is easily scheduled as an early morning appointment for vulnerable patients.

6. Curbside check-in is available to decrease time in a waiting room at some imaging centers.

7. Shorter procedural time (15-20 $\mathrm{min})$, with no need for pre-procedure IV placement or post-procedure monitoring reduces time in a healthcare facility.

8. Less direct contact with fewer health care workers (e.g., one CT technologist and/or one radiologist is intermittently in the CT suite during the exam).

9. Decreased need for personal protective compared to optical colonoscopy as more healthcare workers are required for optical colonoscopy (e.g., endoscopist, anesthesiologist or nurse anesthetist, nurse(s) for IV placement and procedural and post-procedural monitoring).

10. CTC can better triage patients for polypectomy or surveillance depending on lesions found as compared to stool-based tests and can better detect precancerous lesions as compared to stool-based tests.

stool specimens tested positive for COVID-19 with rRTPCR testing [8]. In a meta-analysis, $17.6 \%$ of patients with COVID-19 had gastrointestinal symptoms, and COVID-19 viral DNA was detected in stool samples in $38.5 \%$ of patients with diarrhea and $8.7 \%$ of patients without diarrhea [22]. COVID-19 has also been found to linger in stool longer than in respiratory or serum samples, a median of 22 days in one study [7]. COVID-19 is thought to enter host cells via cell receptor angiotensin converting enzyme II (ACE2) [23]. ACE2 is highly expressed in lung cells as well as those in the esophagus, ileum, and colon which may account for its gastrointestinal manifestations [23, 24].

Symptomatic acute colitis is a relative contraindication to performing CTC [25]. As abdominal pain and diarrhea can be symptoms of COVID-19, screening CTC should not be performed in symptomatic patients. Some institutions may require a negative COVID-19 test prior to CTC performance. Although elective screening CTC should not be performed in patients thought to be infected with COVID-19, it is known that many patients with COVID-19 are asymptomatic and the presence of characteristic nodular, peripheral ground glass opacities with a lower lobe predominance in the lung bases at CTC could indicate a viral pneumonia, possibly caused by SARS-CoV2 and necessitates appropriate communication with the referring provider [26].

Whether or not fecal transmission of COVID-19 occurs is unknown. Current guidance from the Centers for Disease Control and Prevention states that "there has not been any confirmed report of virus spreading from feces to a person", and the risk of fecal transmission is thought to be low based on data from previous disease outbreaks caused by other related coronaviruses including Middle East respiratory syndrome (MERS) and severe acute respiratory syndrome (SARS) [27]. As CTC is a theoretical aerosol generating procedure (along with esophagrams, upper GI fluoroscopic studies, and contrast enemas), until more is known about fecal transmission, CTC should be performed with PPE as recommended by institutional guidelines for potential aerosol generating procedures [28].

\section{Conclusion}

Postponement of cancer screening due to COVID-19 has resulted in a backlog of individuals needing to undergo structural examination of the colon. The experience during the initial surge with urgent evaluation of the colon for transplant patients prior to transplant suggests that CTC can be done in a lower risk manner. The procedural profile of CTC is advantageous during this pandemic as maintaining social distancing and preserving healthcare supplies including PPE are of paramount importance. CTC is an important option to utilize in the screening armamentarium to allow effective screening of average risk asymptomatic individuals in the COVID-19 era.

\section{Compliance with ethical standards}

Conflict of interest Ahmed, Brewington, Chang, Dachman, Gollub, McFarland, Reddy, Zalis, Yee: None. Barish: Stockholder, Blackford Analysis; Consultant, Parexel Inc; Consultant, American College of Radiology. Kim: Shareholder in Elucent and Cellectar. Moreno: Research agreement GE Healthcare. Pickhardt: Advisor to Bracco and Zebra; Shareholder in SHINE and Elucent. 


\section{References}

1. Epic Health Research Network. Preventive Cancer Screenings during COVID-19 Pandemic. https://ehrn.org/wp-content/uploa ds/Preventive-Cancer-Screenings-during-COVID-19-Pandemic. pdf (Accessed May 6, 2020)

2. Centers for Disease Control and Prevention. Healthcare Facilities: Preparing for Community Transmission. 2/29/2020 (Accessed April 25, 2020)

3. American Cancer Society. Common Questions About the New Coronavirus Outbreak. https://www.cancer.org/latest-news/ common-questions-about-the-new-coronavirus-outbreak. html\#shouldpeoplestillgetscreenedforcancerduringthispandemic (Accessed May 6, 2020)

4. Centers for Medicare and Medicaid Services. Opening Up America Again: Centers for Medicare \& Medicaid Services (CMS) Recommendations Re-opening Facilities to Provide Non-emergent Non-COVID-19 Healthcare: Phase 1. https:// www.cms.gov/files/document/covid-flexibility-reopen-essen tial-non-covid-services.pdf (Accessed April 25, 2020)

5. Sun LH. CDC director warns second wave of coronavirus is likely to be even more devastating. The Washington Post. April 21,2020

6. American Society for Gastrointestinal Endoscopy. Gastroenterology professional society guidance on endoscopic procedures during the COVID-19 pandemic. https://www.asge.org/home/ advanced-education-training/covid-19-asge-updates-for-membe rs/gastroenterology-professional-society-guidance-on-endos copic-procedures-during-the-covid-19-pandemic (Accessed April 25, 2020)

7. Zheng S, Fan J, Yu F, et al. Viral load dynamics in disease severity in patients infected with SARS-CoV-2 in Zhejiang province, China, January-March 2020; retrospective cohort study. BMJ. 2020 Apr 21;369:m1443. doi: https://doi.org/10.1136/bmj.m1443.

8. Holshue ML, DeBolt C, Lindquist S, et al. First Case of 2019 Novel Coronavirus in the United States. N Engl J Med 2020;382:929-936.

9. Bellini D, Rengo M, De Cecco CN, Iafrate F, Hassan C, Laghi A. Perforation rate in CT colonography: a systematic review of the literature and meta-analysis. Eur Radiol 2014;24:1487-1496.

10. Arora G, Mannalithara A, Singh G, Gerson LB, Triadafilopoulos G. Risk of perforation from a colonoscopy in adults: a large population-based study. Gastrointest Endosc 2009;69:654-664.

11. Warren JL, Klabunde CN, Mariotto AB, et al. Adverse events after outpatient colonoscopy in the Medicare population. Ann Intern Med 2009;150:849-857.

12. Ranasinghe I, Parzynski CS, Searfoss R, et al. Differences in colonoscopy quality among facilities: development of a postcolonoscopy risk-standardized rate of unplanned hospital visits. Gastroenterology 2016;150:103-113.

13. Kim DH, Pickhardt PJ, Taylor AJ, et al. CT colonography versus colonoscopy for the detection of advanced neoplasia. N Engl J Med 2007;357:1403-1412.
14. Moreno CC, Fibus TF, Krupinski EA, Kim DH, Pickhardt PJ. Addressing racial disparity in colorectal cancer screening with CT colonography: experience in an African-American cohort. Clin Colorectal Cancer 2018;17:e363-e367.

15. Zalis ME, Barish, MA, Choi JR, et al. CT Colonography Reporting and Data System: A Consensus Proposal. Radiology 2005;236:3-9.

16. Imperiale TF, Ransohoff DF, Itzkowitz SH, et al. Multitarget stool DNA testing for colorectal-cancer screening. N Engl J Med 2014;370:1287-1297.

17. Moreno CC, Duszak R, Yee J, Horny M. Geographic dispersion and rural versus urban utilization of CT colonography in the United States. J Am Coll Radiol 2020;17:475-483.

18. https://www.acr.org/Clinical-Resources/Colon-Cancer-ScreeningResources/My-CT-Colonography (Accessed April 25, 2020)

19. Koh HK, Sebelius KG. Promoting prevention through the Affordable Care Act. N Engl J Med 2010;363:1296-1299.

20. US Preventive Services Task Force. Screening for Colorectal Cancer: US Preventive Services Task Force Recommendation Statement. JAMA 2016;315:2564-2575.

21. Sawhney TG, Pyenson BS, Rotter D, Berrios M, Yee J. Computed tomography colonography less costly than colonoscopy for colorectal cancer screening of commercially insured patients. Am Health Drug Benefits 2018;11:353-361.

22. Cheung KS, Hung IFN, Chan PPT, et al. Gastrointestinal manifestations of SARS-CoV-2 infection and virus load in fecal samples from the Hong Kong Cohort and Systematic Review and MetaAnalysis. Gastroenterology

23. Zhang H, Kang Z, Gong H, et al. The digestive system is a potential rout of 2019-nCov infection: a bioinformatics analysis based on single-cell transcriptomes doi: https://doi. org/10.1101/2020.01.30.927806

24. Lamers MM, Beumer J, van der Vaart J, et al. SARS-CoV-2 productively infects human gut enterocytes. Science 2020 May 1. pii: eabc1669. doi: https://doi.org/10.1126/science.abc1669.

25. ACR-SAR-SCBT-MR Practice Parameter for the Performance of Computed Tomography (CT) Colonography in Adults. Revised 2019.

26. Siegel A, Chang PJ, Jarou ZJ, et al. Lung base findings of Coronavirus Disease (COVID-19) on abdominal CT in patients with predominant gastrointestinal symptoms. AJR Am J Roentgenol 2020 Apr 17:1-3. doi: https://doi.org/10.2214/AJR.20.23232

27. Centers for Disease Control and Prevention. Water and COVID-19 FAQs Information about Drinking Water, Treated Recreational Water, and Wastewater. https://www.cdc.gov/coronavirus/2019ncov/php/water.html (Accessed April 25, 2020)

28. Mossa-Basha M, Azadi J, Ko J, Klein J, Meltzer C, COVID-19 Task Force. RSNA COVID-19 Task Force: Best Practices for Radiology Departments during COVID-19. April 27, 2020 https:// www.rsna.org/-/media/Files/RSNA/Covid-19/RSNA-COVID-19bestpractices. ashx?la=en\&hash=58700DDDEDB3E5A9C8ED E80BE534B4ABB10291B7 (Accessed May 5, 2020)

Publisher's Note Springer Nature remains neutral with regard to jurisdictional claims in published maps and institutional affiliations. 